\title{
THE INFLUENCE OF DRINKING FLUID ON ENDURANCE CYCLING
}

\section{PERFORMANCE: A META-ANALYSIS}

Justin J. Holland ${ }^{1,2}$, Tina L. Skinner ${ }^{1}$, Christopher G. Irwin ${ }^{3}$, Michael D. Leveritt ${ }^{1}$ and Eric D.B.

Goulet $^{4,5}$

${ }^{1}$ School of Human Movement and Nutrition Sciences, The University of Queensland, St Lucia, Queensland, Australia

${ }^{2}$ School of Exercise and Nutrition Sciences, Queensland University of Technology, Kelvin Grove, Queensland, Australia

${ }^{3}$ School of Allied Health Sciences, Menzies Health Institute Queensland, Griffith University, Gold Coast, Queensland, Australia

${ }^{4}$ Faculty of Physical Activity Sciences, University of Sherbrooke, Sherbrooke, Québec, Canada

${ }^{5}$ Research Centre on Aging, University of Sherbrooke, Sherbrooke, Québec, Canada

Running Title: Influence of drinking fluid on endurance cycling performance.

Word count: 5751

\section{Correspondence:}

Mr. Justin Holland

School of Exercise and Nutrition Sciences

Queensland University of Technology

O Block, A wing, Victoria Park Road

Kelvin Grove

Queensland 4059

Australia

E-mail: Justin.holland@qut.edu.au 


\section{Key Points}

- The primary finding of this meta-analysis is that the effect of fluid consumption on endurance cycling performance (ECP) is complex and needs to be considered in the context of the exercise task and its associated demands.

- $\quad$ Exercise duration was identified as a key factor that distinguished between the positive and negative effects of fluid ingestion on ECP. During $1 \mathrm{~h}$ high intensity exercise, ECP decreased by $2.5 \%$ when fluid was consumed at high rates $(0.15-0.34 \mathrm{ml} / \mathrm{kg}$ body mass/min), compared to no fluid intake. However, performance improvements of $2.1 \%$ and 3.2\% were observed when fluid was consumed during moderate intensity cycling exercise of $>1-\leq 2 \mathrm{~h}$ and $>2 \mathrm{~h}$ duration, respectively.

- $\quad$ To optimise ECP, cyclists should avoid consuming fluids at a rate between $0.15-0.34 \mathrm{ml} / \mathrm{kg}$ body mass/min during high intensity exercise of $1 \mathrm{~h}$, but should consume $0.15-0.20 \mathrm{~mL} / \mathrm{kg}$ body mass/min of fluid during exercise of $>1-\leq 2 \mathrm{~h}$ and drink ad libitum or consume 0.14$0.27 \mathrm{~mL} / \mathrm{kg}$ body mass $/ \mathrm{min}$ for exercise duration $>2 \mathrm{~h}$. 


\begin{abstract}
BACKGROUND: Fluid replacement during cycling exercise evolves on a spectrum from simply drinking to thirst to planned structured intake, with both being appropriate recommendations. However, with mixed findings suggesting fluid intake may or may not improve endurance cycling performance (ECP) in a diverse range of trained individuals, there is clear need for summarised evidence regarding the effect of fluid consumption on ECP.
\end{abstract}

OBJECTIVES: 1) Determine the magnitude of the effect of drinking fluid on performance during cycling exercise tasks of various durations, compared with no drinking; 2) examine the relationship between rates of fluid intake and ECP and; 3) establish fluid intake recommendations based on the observations between rates of fluid intake and ECP.

STUDY DESIGN: Meta-analysis.

METHODS: Studies were located via database searches and cross-referencing. Performance outcomes were converted to a similar metric to represent \% change in power output. Fixed- and random-effects weighted mean effect summaries and meta-regression analyses were used to identify the impact of drinking fluid on ECP.

RESULTS: A limited number of research manuscripts $(n=9)$ met the inclusion criteria, producing 15 effect estimates. Meta-regression analyses demonstrated that the impact of drinking on ECP under $20-33^{\circ} \mathrm{C}$ ambient temperatures was duration-dependent. Fluid consumption of on average 0.29 $\mathrm{mL} / \mathrm{kg}$ body mass/min impaired $1 \mathrm{~h}$ high intensity ( $\left.80 \% \dot{\mathrm{V}}_{2 \text { peak }}\right)$ ECP by $-2.5 \pm 0.8 \%$ (95\% CI: 4.1- $-0.9 \%)$, compared to no fluid ingestion. In contrast, during $>1-\leq 2 \mathrm{~h}$ and $>2 \mathrm{~h}$ moderate intensity $\left(60-70 \% \dot{\mathrm{V}}_{2 \text { peak }}\right)$ cycling exercise, ECP improved by $2.1 \pm 1.5 \%$ (95\% CI: $\left.1.2-2.9 \%\right)$ and $3.2 \pm$ 1.2\% (95\% CI: 0.8-5.6\%), respectively, with fluid ingestion compared to no fluid intake. The associated performance benefits were observed when the rates of fluid intake were in the range of 0.15-0.20 mL/kg body mass/min for $>1-\leq 2 \mathrm{~h}$ cycling exercise and ad libitum or $0.14-0.27 \mathrm{~mL} / \mathrm{kg}$ body mass/min for cycling exercise $>2 \mathrm{~h}$. 
CONCLUSIONS: A rate of fluid consumption between $0.15-0.34 \mathrm{ml} / \mathrm{kg}$ body mass/min during high intensity $1 \mathrm{~h}$ cycling exercise is associated with reductions in ECP. When cycling at moderate intensity for $>1-\leq 2 \mathrm{~h}$, cyclists should expect a gain in performance of at least $2 \%$ if fluid is consumed at a rate of $0.15-0.20 \mathrm{~mL} / \mathrm{kg}$ body mass $/ \mathrm{min}$. For cycling exercise $>2 \mathrm{~h}$ conducted at moderate intensity, consuming fluid ad libitum or at a rate of $0.14-0.27 \mathrm{~mL} / \mathrm{kg}$ body mass $/ \mathrm{min}$ should improve performance by at least 3\%. Until further research is conducted, these recommendations should be used as a guide to inform hydration practices. 


\section{INTRODUCTION}

Goulet [1, 2] meta-analytically identified that dehydration of up to $4 \%$ body mass loss does not impair endurance cycling performance (ECP). However, based on the numerous physiological and psychological benefits of fluid ingestion during exercise, it is unreasonable to expect cyclists not to consume fluid during an event where anticipated dehydration will be less than $4 \%$ of body mass. In fact, consuming fluid during cycling exercise offers benefits of lower perceived exertion, decreased thirst sensation, reduced cardiovascular and thermal stress and enhanced substrate utilization, compared to no fluid ingestion [3, 4]. Moreover, stimulation of the pharyngeal receptors with a trivial amount of fluid ingestion $(100 \mathrm{~mL})$ has been shown to improve endurance cycling capacity, compared with mouth rinsing only or no fluid intake [5]. The authors speculated that the act of swallowing fluid and the subsequent cooling sensation in the digestive tract may be enough of a motivational factor to promote improved performance. Independent of the causal factor(s), this observation suggests that the simple act of drinking fluid during cycling exercise may benefit performance and that the perception of drinking fluid may be as, if not more, important for athletes than the absolute volume consumed during exercise.

The current American College of Sports Medicine (ACSM) Position Stand on exercise and fluid replacement highlights that the amount and rate of fluid replacement depends upon the individual sweating rate, exercise duration and opportunities to drink [3]. Additionally, the guidelines suggest that longer exercise durations lead to a disproportionate balance between fluid replacement and requirements leading to dehydration [3]. This is also indicated in endurance cycling tasks that are $>1 \mathrm{~h}$ with a stronger negative association between changes in cycling power output and exercise duration in comparison to exercise-induced dehydration [1]. Thus, with the potential for ECP to be relatively well maintained despite a mismatch between rates of fluid intake and sweat losses during cycling exercise durations $<1 \mathrm{~h}$ [6-8], but not necessarily during longer exercise [9, 10], it is plausible that fluid ingestion may offer an ECP benefit which is duration-dependent. To the best of our knowledge, no meta-analysis has yet attempted to quantify the effect of fluid ingestion on 
performance during cycling tasks of various durations. Moreover, we are not aware of any metaanalysis which has evaluated whether a possible relationship between rates of fluid intake and ECP exists.

Strategies for fluid replacement during cycling exercise are comprised within a large spectrum and may range from purely relying on thirst sensation [11] to customized fluid ingestion plans based on the estimation of sweat losses, usually with the aim of preventing a body mass loss $>2 \%$ [3]. A major limitation of the reliance on thirst sensation to gauge fluid intake is that under certain circumstances (e.g., psychological stress, repeated acute food intake, cold temperature, various medications, older athletes) [11] thirst may not be a reliable indicator of fluid needs, which may lead to suboptimal fluid intake and ECP. Moreover, some individuals may simply be reluctant to rely on thirst-related sensations to guide their intake of fluid during cycling exercise [11]. On the other hand, customized individual plans require athletes to estimate sweat losses during a particular cycling task with respect to exercise intensity and environmental conditions. However, this may not always be practical, as underlined by the ACSM [3]. Given these limitations, it would helpful for those individuals to be able to rely on evidence-based, practical recommendations providing guidance on the optimal rates of fluid intake during cycling exercise of various durations.

The goal of this meta-analysis was threefold: 1) determine the magnitude of the effect of drinking fluid on performance during cycling exercise of various durations; 2) examine the relationship between rates of fluid intake and ECP and; 3) provide guidelines on the optimal rates at which cyclists should be drinking while exercising to optimize performance. The results of the present meta-analysis will assist coaches, cyclists and health professionals regarding optimal rates of fluid intake during cycling exercise.

\section{METHODS}

\subsection{Data sources}

The process used for selecting research articles is outlined as a process flow chart in Figure 1. A systematic search of the PubMed (MEDLINE) (http://www.ncbi.nlm.nih.gov/pubmed), Scopus 
(http://www.scopus.com/) and Web of Science (http://apps.webofknowledge.com/) online databases with no date limitation was conducted using the following keywords (alone or in combination): hydration, dehydration, hypohydration, rehydration, hyperhydration, fluid balance, fluid consumption, endurance performance, exercise capacity and exercise performance. Articles that pertained to fluid ingestion and ECP were identified. When a potential article was found, the title and abstract were first read by the first author. If the abstract revealed that an intervention was made to determine the effect of exercise-induced hypohydration and/or fluid ingestion on ECP, then the entire article was read by the first author to determine eligibility. In the event of inclusion discrepancies, they were resolved by a review from the third and fourth authors. Reference lists from retrieved articles, as well as published articles [2] were further examined to identify additional articles for potential inclusion. Case studies, conference proceedings, published abstracts, dissertations and manuscripts published in non-peer-reviewed journals were excluded. Only English-written articles were considered during the research process. The last day of the search was performed on 11 April 2015. The Preferred Reporting Items for Systematic Reviews and MetaAnalysis guidelines were followed during the preparation of this review and presentation of the results [12].

\subsection{Inclusion and exclusion criteria}

The following inclusion criteria were used to identify articles qualifying for this meta-analysis: 1) participants were healthy adults (men or women) aged $\geq 18$ years; 2 ) the study included a control group that did not drink during exercise; 3) fluid replacement was given orally; 4) hypohydration was induced during, not before exercise; 5) the study was laboratory-controlled and cycling-related; 6) the performance measure involved continuous cycling exercise $>2$ min; 7) data needed to calculate \% changes in power outputs, effect estimates and variances were provided; 8) \% body mass losses were reported and; 9) nutrient intake was matched between experimental groups. An article was excluded if it presented with any of the following: 1) no measured performance outcome and; 2) participants were unable to complete the entire exercise protocol, as a priori defined. 


\subsection{Data extraction}

Coding sheets with operational definitions were developed and utilized for this investigation. When necessary, authors were contacted to resolve ambiguities and issues with methodology or findings. Coded variables included: 1) study characteristics; 2) participant physical and fitness characteristics; 3) exercise protocol characteristics; 4) fluid intakes during exercise and hypohydration levels and; 5) exercise performance.

\subsection{Exercise protocol characteristics}

Exercise duration represents the mean total exercise time (min) completed in the no fluid intake and fluid intake groups [1]. Exercise intensity represents the mean relative peak oxygen consumption ( $\left.\mathrm{V}_{2 \text { peak }}\right)$ at which the exercise protocols (i.e., no fluid and fluid intake groups) were conducted. A weighted average technique was used to determine exercise intensity of studies that combined multiple bouts of exercise conducted at different intensities. Studies by Dugas et al. [9] and Kay and Marino [7] did not indicate the percent of $\dot{\mathrm{vo}}_{2 \text { peak }}$ at which the exercise protocols were conducted, therefore exercise intensity was computed as explained by Goulet [2].

\subsection{Fluid consumption and hypohydration level}

Fluid consumption represents the entire amount of fluid consumed over the total duration of exercise. Any fluid consumed within the 5 min period prior to the start of exercise was included in the computation of total fluid consumption, as this fluid was integrated into the body during exercise and contributed to physiological regulation $[13,14]$. The rate of fluid consumption $(\mathrm{mL} / \mathrm{kg}$ body mass/min) was calculated by dividing the overall fluid consumed (mL) during a given exercise trial by pre-exercise body mass (kg) and then by the total exercise time (min). Hypohydration level was taken as the \% change in body mass from pre- to post-exercise. It is acknowledged that assessment of hypohydration using changes in body mass lacks precision. However, under field conditions, it is the most practical and reliable method of estimating hypohydration level [15].

\subsection{Exercise performance}


Five studies [6-9, 13] used a time-trial type exercise protocol to test ECP, with all reporting mean maintained power output during exercise. Hence, in these studies, the \% change in cycling performance was measured using the following formula:

Eq 1.: ((Fluid intake group mean power output - no fluid intake group mean power output) / no fluid intake group mean power output) x 100 .

Four studies[10, 14, 16, 17] used a fixed-power output test to exhaustion to evaluate the impact of fluid intake on ECP. To make results comparable among all studies included in this meta-analysis, the \% changes in endurance cycling capacity were transformed to equivalent changes in performance during a time-trial type exercise, according to Equation 2:[2]

Eq 2.: (((Fluid intake group mean time to exhaustion - no fluid intake group mean time to exhaustion) / no fluid intake group mean time to exhaustion) x 100) / (\% $\dot{\mathrm{V}}_{\text {O2peak }}$ at which the test was performed / 6.4)

\subsection{Statistical Analysis}

Data were analyzed using Microsoft Office Excel 2007 (version 12.0.6735.5000, Redmond, WA, USA), IBM SPSS Statistics (version 21.0.0.0, Armonk, NY, USA) and Comprehensive MetaAnalysis (CMA; version 2.2.064, Englewood, NJ, USA) softwares along with SPSS macros developed by Lipsey and Wilson [18].

\subsubsection{Weighted mean effect summary}

A weighted mean effect summary including $>10$ studies was computed using a random-effects model, whereas when the number of studies was $\leq 10$ it was computed using a fixed-effect model. This procedure was followed since when the number of studies is small the estimate of the betweenstudy variance to be used with a random-effects model has poor precision. When possible, variances were directly calculated from the reported $\Delta$ standard errors or standard deviations of the net percent changes in cycling performance. When differences between groups were not reported, variances were computed from reported P values or confidence intervals (CI) and, when neither were reported, from $\mathrm{P}$ values equal to $X$, where $X$ is any $\mathrm{P}$ values $\leq 0.05$.[19] When only $\mathrm{P}>0.05$ 
was reported, individual variances for net percent changes in cycling performance were estimated with the formula used by the CMA software, using an imputed correlation coefficient [19] of 0.68 computed from the raw experimental results of six individual studies provided by two researchers [8, 9]. Several research articles included more than one treatment effect. To account for independency of research data, two separate statistical analyses were performed where, on one occasion, the weighted mean effect summary was determined with only one effect estimate and weighting factor per research article (representing the mean effect estimate and weighting factor of all studies included in the research article) and on the other, where each outcome was treated independently. If both approaches yielded similar figures, then the model treating each outcome independently was retained since it provides a greater level of information. Qualitative interpretation of the practical significance of the effect of fluid intake on cycling performance under real-world conditions was computed using the spreadsheet developed by Hopkins [20]. The smallest worthwhile \% change in power output was determined for long-distance cyclists and set at $1.6 \%$ [1]. For each performance outcome, the normality of data distribution was verified using the Shapiro-Wilk test. Results are reported as means \pm standard errors.

\subsubsection{Evaluation of heterogeneity and publication bias}

Heterogeneity and publication bias were determined only when a weighted mean effect summary was derived from $>10$ studies. Between-study heterogeneity was assessed using the $I^{2}$ statistic and the Cochran $Q$ test, with $\mathrm{P} \leq 0.01$ indicating significance [21]. Whether there was evidence of literature bias was examined with a funnel plot visual inspection and statistically tested using Egger's test of the intercept [21]. The Orwin's fail-safe N procedure was used to test if the overall observed weighted mean treatment effect was an artifact of bias [21], with the smallest worthwhile $\%$ change in power output brought about by drinking taken as $1.6 \%$ [2]. Finally, the potential impact of bias was estimated using the trim and fill technique [21].

\subsubsection{Meta-regression analysis}


Meta-regressions including $>10$ data points were performed using a restricted maximum likelihood random-effects model. When the number of studies was $\leq 10$, they were computed using a fixedeffect model. Categorical variables were dummy-transformed with $k-1$, where $\mathrm{k}$ is the number of levels of the original variable. Multiple regression analyses were examined for the presence of multicollinearity between predictor variables (variance inflation factor). The alpha level for statistical significance was set at $\mathrm{P} \leq 0.05$.

\section{RESULTS}

\subsection{Search results}

The initial database search identified 15532 unique records. After screening the titles, a total of 483 articles were identified that were potentially related to the topic of investigation and their abstracts were subsequently read. Of these, 151 potentially matched the inclusion criteria and full-text articles were read. Nine [6-10, 13, 14, 16, 17] met all inclusion criteria. Figure 1 details the search procedure and reasons for exclusion after full-text extraction. Two separate studies were performed within McConell et al. [10], McConell et al. [8] and Kay and Marino [7], whilst four separate studies were included within Dugas et al. [9], yielding a total of 15 individual studies. Table 1 provides a detailed description of participant characteristics, exercise protocols and rates of fluid intake and hypohydration levels of each included research manuscript and individual study, which were classified according to exercise duration, i.e., $1 \mathrm{~h},>1-\leq 2 \mathrm{~h}$ and $>2 \mathrm{~h}$.

\subsection{Characteristics of the research manuscripts}

The included manuscripts were published between 1989 and 2011 in seven different peer-reviewed journals. One investigation was published in the 1980s [17], five between the years of 1990 and $1999[8,10,13,14,16]$ and three between 2000 and 2010 [6, 7, 9]. Three investigations were performed in Australia $[7,8,10]$ and South Africa $[9,13,14]$, two in the UK $[16,17]$ and one in the USA [6].

\subsection{Description of participants}


A total of 70 participants were represented in the nine research manuscripts sourced. The average sample size per study was $7 \pm 2$ participants. Women represented $10 \%$ of the participants across the 15 studies. The average age, height, body mass and relative $\dot{\mathrm{v}}_{2 \text { peak }}$ of participants was $25 \pm 3$ years, $1.77 \pm 0.04 \mathrm{~m}$ ( $\mathrm{n}=8$ research manuscripts), $72 \pm 5 \mathrm{~kg}$ and $61 \pm 7 \mathrm{~mL} / \mathrm{kg}$ body mass $/ \mathrm{min}(\mathrm{n}=6$ research manuscripts), respectively. None of the studies provided data on ethnicity.

\subsection{Exercise protocol characteristics}

\subsubsection{Time-trial protocols}

Four studies utilized a time-trial protocol where participants had to cover the greatest possible distance in a given time $[6,7,13]$. The remaining studies $(n=4)$ used a fixed-distance cycling timetrial [9].

3.4.2 Continuous and fixed-power output protocols

Two studies used a continuous, fixed-power output test to exhaustion conducted at the same relative exercise intensity throughout $[16,17]$. Three studies used a continuous exercise protocol where an initial fixed power output bout of exercise was followed by another one at a higher intensity [10, 14]. Two studies used a continuous exercise protocol where a first fixed-power output exercise period was followed by one where the participants had to perform the greatest amount of work possible in a designated amount of time [8].

3.4.3 Environmental conditions, exercise intensity and exercise duration

The mean ambient temperature, relative humidity, exercise intensity and exercise duration observed in the 15 studies combined was $22 \pm 6^{\circ} \mathrm{C}, 50 \pm 12 \%, 69 \pm 12 \%$ of $\dot{\mathrm{v}} \mathrm{O}_{2 \text { peak }}$ and $69 \pm 12 \mathrm{~min}$, respectively (Table 1).

\subsection{Fluid consumption and hypohydration level}

Overall, the mean total fluid consumption for the 15 studies included was $1400 \pm 556 \mathrm{~mL}(16 \pm 6$ $\mathrm{mL} / \mathrm{min}$ ), with a rate of fluid intake based on body mass of $0.23 \pm 0.08 \mathrm{~mL} / \mathrm{kg} / \mathrm{min}$. Hypohydration level for the no fluid intake group reached $-2.7 \pm 1.1 \%$ of body mass, whereas the fluid intake group 
attained $-0.8 \% \pm 1.0 \%$ of body mass. Mean rates of fluid intake and hypohydration levels based on exercise duration classification are reported in Table 1.

\subsection{Exercise performance}

3.6.1 Overall weighted mean effect summary

The impact of fluid intake on ECP is shown in Figure 2. These data were normally distributed. Drinking fluid during exercise did not significantly improve or impair cycling performance (1.08 \pm 0.91\%, 95\% CI: $-0.7-2.9 \%$; $\mathrm{P}=0.23$ ) based on 15 comparisons from nine studies. Within this model, the impact of fluid intake on ECP was considered trivial. A computation using only one finding per research article yielded an effect of drinking on cycling performance of $0.9 \pm 0.9 \%$ (95\% CI: $-0.9-2.8 \%, \mathrm{P}=0.32$ ). The magnitude of this effect was no different from that determined when all individual studies were considered to be independent $(P=0.93)$. A sensitivity analysis demonstrated that deletion of each study one at a time from the model changed neither the magnitude nor the significance of the impact of fluid intake on ECP.

\subsubsection{Heterogeneity}

Visual examination of Figure 2 suggested a lack of homogeneity of the effect of fluid intake on ECP, which was statistically confirmed by a $Q$ of $49.5(\mathrm{P}<0.01)$ and an $I^{2}$ value of $72 \%$ (high heterogeneity). The widely different responses to the effect of drinking during exercise, explains in part the trivial and non-significant change in ECP reported in Figure 2.

\subsubsection{Publication bias}

Inspection of the funnel plot (figure not shown) suggested no significant publication bias. Moreover, the Egger's test of the intercept $(-0.10 \pm 0.82 \%)$ was not statistically significant $(\mathrm{P}=$ 0.45). There would need to be 14 unpublished studies with a mean change in ECP of $2 \%$ due to fluid intake before the weighted mean treatment effect would become practically significant and relevant (i.e., $>1.6 \%$ ). Based on a left trim and fill correction of two additional studies, the potential impact of publication bias would be to reduce the effect of drinking on ECP by $0.62 \%$, down to $0.46 \%$ (95\% CI: $-1.38-2.30 \%)$. 


\subsubsection{Meta-regression analyses}

Figure 3 shows the relationships between the change in ECP and the rate of fluid intake during exercise (a), exercise duration (b) and exercise intensity (c). There was no significant correlation between the rate of fluid intake during exercise and the change in ECP ( $\mathrm{P}=0.73)$. However, significant relationships were observed between the change in ECP and exercise duration $(\mathrm{P}=0.03)$ (b) and intensity $(\mathrm{P}=0.03)(\mathrm{c})$. Figure 4 demonstrates that there was no significant correlation between the change in power output and ambient temperature (a) $(\mathrm{P}=0.10)$ or humidity level (b) (P $=0.78)$. The impact of drinking on ECP in studies using continuous, fixed-power output $(0.6 \pm$ $1.7 \%) v s$. time-trial $(1.5 \pm 1.3 \%)$ exercise protocols was not significantly different $(\mathrm{P}=0.67)$.

There was a significant $(\mathrm{P}<0.01)$ and relatively strong $\left(\mathrm{R}^{2}=64 \%\right)$ correlation between exercise duration and intensity (results not shown). However, when both variables were combined in the same model, exercise intensity did not provide any additional information beyond that supplied by exercise duration. Since exercise duration correlated with the rate of fluid intake during exercise, we determined if the rate of fluid intake correlated with the change in power output while controlling for the effect of exercise duration. In that respect, Figure 5 shows that there was a significant correlation between the rate of fluid intake during exercise and the change in power output for cycling $>1-\leq 2 \mathrm{~h}(\mathrm{P}<0.01)$ and $>2 \mathrm{~h}(\mathrm{P}<0.01)$ in duration, but not when exercise was $1 \mathrm{~h}$ duration $(\mathrm{P}$ $=0.76)$.

As demonstrated in Figure 6a, there was a significant positive correlation between the capacity of fluid intake to prevent the loss of body mass and change in power output $(\mathrm{P}<0.01)$. Figure $6 \mathrm{~b}$ demonstrates that this relationship was dependent upon exercise duration, since the hypohydration level observed in the fluid intake group was correlated with the change in power output for cycling exercise of $>1-\leq 2 \mathrm{~h}(\mathrm{P}<0.01)$ and $>2 \mathrm{~h}(\mathrm{P}=0.01)$ duration, but not when exercise was $1 \mathrm{~h}$ duration $(\mathrm{P}=0.58)$. 
The above findings indicate that the impact of fluid intake and associated hypohydration level on ECP is exercise duration-dependent. Based on this observation, the following section reports the impact of fluid intake on ECP for exercise duration of $1 \mathrm{~h},>1-\leq 2 \mathrm{~h}$ and $>2 \mathrm{~h}$.

3.6.5 Weighted mean effect summary based on exercise duration

Figure 7 reports the impact of fluid intake on ECP during exercise of $1 \mathrm{~h}(\mathrm{a}),>1-\leq 2 \mathrm{~h}$ (b) and $>2 \mathrm{~h}$ (c) in duration. All performance outcomes were normally distributed. Whether all studies were considered independently $(-2.5 \pm 0.8 \%, 95 \%$ CI: $-4.1--0.1 \%)$ or when only one study per research manuscript was included in the model $(-2.7 \pm 1.1 \%$, 95\% CI: $-4.8--0.5 \%)$, drinking during exercise of $1 \mathrm{~h}$ duration resulted in a significant reduction of ECP. The impact of drinking during $1 \mathrm{~h}-$ long cycling exercise is likely important. The outcome was not different using a random-effects weighted mean effect summary. A sensitivity analysis demonstrated that deletion of each study one at a time from the model changed neither the magnitude nor the significance of the impact of fluid intake on ECP. On the other hand, when exercise duration was $>1-\leq 2 \mathrm{~h}(2.1 \pm 1.5 \%, 95 \%$ CI: $1.2-2.9 \%)$ (random-effects model: $2.0 \pm 0.8 \%, \mathrm{P}=0.02)$ or $>2$ h $(3.2 \pm 1.2 \%, 95 \%$ CI: $0.8-5.6 \%)$ (randomeffects model: $4.2 \pm 2.3 \%, \mathrm{P}=0.06$ ), ECP was significantly improved by drinking fluid. The impact of fluid intake on ECP under real-world conditions is likely or very likely to be important ( $>1.6 \%$ meaningful change) [2]. When dependency among studies was taken into account during cycling $>2$ $\mathrm{h}$ in duration, drinking tended $(\mathrm{P}=0.052)$ to benefit ECP and increased power output by $4.7 \pm 2.4 \%$ (95\% CI: $-0.0-9.4 \%$ ). For exercise duration $>1-\leq 2 \mathrm{~h}$, a sensitivity analysis indicated that deleting each study one at a time from the model did not substantially alter the magnitude of the effect of fluid intake on ECP. However, when the study of Maughan et al. [16] was removed from the summary, the effect became non significant. For cycling exercise $>2 \mathrm{~h}$ in duration, removing the study (c) of Dugas et al. [9] from the model doubled the effect of fluid intake on ECP. Removal of any other studies did not change the magnitude of the effect of drinking on ECP, but deleting that (a) of Dugas et al. [9] or (a) McConell et al. [10] removed significance. 
3.6.6 Optimal rates of fluid intake based on exercise duration

\subsubsection{Exercise of $1 \mathrm{~h}$}

Figure 8a demonstrates that with the exception of one study [6] where fluid intake proved beneficial to ECP, fluid intake in the range of $0.15-0.34 \mathrm{~mL} / \mathrm{kg}$ body mass $/ \mathrm{min}$ either had no impact or decreased power output, despite preventing $>2 \%$ loss of body mass. That was true for cycling exercise performed in temperate [7, 8, 13] and warm ambient conditions [7] and for continuous, fixed-power output [8] and time-trial [7, 13] exercise protocols.

\subsubsection{Exercise $>1-\leq 2 \mathrm{~h}$}

As depicted in Figure 8b, it appears that fluid intake in the range of $0.15-0.20 \mathrm{~mL} / \mathrm{kg}$ body mass/min has a positive and graded impact on ECP. These rates of fluid intake were sufficient to prevent $>2 \%$ loss of body mass, in temperate $[16,17]$ and warm [14] ambient conditions. Although these findings were all derived from studies that used continuous, fixed-power output exercise protocols, we propose that there is no reason to believe that they could not also apply to out-ofdoors cycling exercise conditions (i.e., road races/time-trials). In fact, the impact of drinking on ECP was similar for both type of exercise protocols during $1 \mathrm{~h}$ (Figure 8a) and $>2 \mathrm{~h}$ (Figure 8c) cycling periods.

\subsubsection{Exercise $>2 \mathrm{~h}$}

As suggested by Figure 8c, the rate of fluid intake associated with the greatest performance improvement during cycling exercise $>2 \mathrm{~h}$ in duration conducted in temperate [10] and warm [9] ambient conditions appears to be between $0.14-0.27 \mathrm{~mL} / \mathrm{kg}$ body mass $/ \mathrm{min}$. These findings applied to both time-trial [9] and continuous, fixed-power output [10] exercise protocols. This model further suggests that drinking above a rate of $0.27 \mathrm{~mL} / \mathrm{kg}$ body mass/min provides no additional increase in cycling performance. The results also showed that a rate of drinking of $0.16 \mathrm{~mL} / \mathrm{kg}$ body mass $/ \mathrm{min}$ consumed ad libitum was linked to the greatest improvement in ECP. Figure 8c shows that drinking at a rate above the dictates of thirst sensation was sufficient to prevent $>2 \%$ loss of body 
mass [10], whereas a rate of drinking according to thirst sensation allowed a loss of body mass slightly greater than $2 \%$ (i.e., $2.1 \%$ body mass) [9].

\section{Discussion}

This is the first meta-analysis to 1) determine the magnitude of the effect of fluid consumption on ECP during tasks of various durations; 2) examine the relationship between rates of fluid intake and ECP and; 3) establish fluid intake recommendations based on the observations made between rates of fluid intake and ECP. The results demonstrated that 1) consuming fluid at rates between 0.15$0.34 \mathrm{~mL} / \mathrm{kg}$ body mass/min during $1 \mathrm{~h}$ high intensity cycling exercise is not advantageous to ECP and; 2) rates of fluid intake of $0.15-0.20 \mathrm{~mL} / \mathrm{kg}$ body mass $/ \mathrm{min}$ and ad libitum or $0.14-0.27 \mathrm{~mL} / \mathrm{kg}$ body mass/min are associated with improved ECP for exercise durations $>1-\leq 2 \mathrm{~h}$ and $>2 \mathrm{~h}$, respectively. With the exception of when fluid was consumed ad libitum, these rates of fluid intake were sufficient to prevent a body mass loss $>2 \%$.

The primary aim of the current meta-analysis was to determine if fluid consumption improves ECP. The overall weighted-mean effect summary indicated no benefit of fluid consumption on ECP, which was attributed to a clear heterogeneity amongst the included studies. Heterogeneity of results, small sample sizes and variations in the cycling task and rate of fluid intake between studies underline the complexity in formulating a practical guideline for fluid intake. Consequently, providing a set fluid prescription and volume as a blanket guideline and replacement strategy clearly does not offer a benefit to cycling performance for all individuals over a range of exercise durations, intensities and environmental conditions.

In certain situations, the rate at which fluid is consumed can be detrimental to ECP, particularly when the exercise is short and of vigorous intensity. Results of the weighted-mean effect summary indicated that structured fluid intake rates ranging from 0.15 to $0.34 \mathrm{~mL} / \mathrm{kg}$ body mass $/ \mathrm{min}$ during high intensity ( $\sim 80 \% \dot{\mathrm{V}}_{2 \text { peak }}$ ) cycling of $1 \mathrm{~h}$ reduced power output by $2.5 \%$, compared to when no fluid is ingested. In fact, in cycling tasks of $1 \mathrm{~h}$ the magnitude of hypohydration was not sufficient to meaningfully perturb physiological homeostasis and power output [6-8, 13]. Interestingly, Backx 
et al. [22] observed that under real-world exercise conditions trained cyclists did not consume fluid during a $40 \mathrm{~km}$ time-trial held in moderate ambient conditions. However, $60 \%$ of the cyclists consumed fluid ( $5 \mathrm{~mL} / \mathrm{kg}$ body mass) during the warm-up period and waiting time leading to the race start, which may have positively influenced ECP. It is interesting, although not surprising, to have observed that consuming large amounts of fluid during $1 \mathrm{~h}$ of vigorous cycling impairs performance. It has been demonstrated that above an exercise intensity of $70-75 \% \dot{\mathrm{V}}_{2 \text { peak }}$ gastric emptying becomes compromised and fluid starts to accumulate in the stomach, leading to abdominal bloating, gastrointestinal discomfort and reduced cycling performance [23, 24]. In the present meta-analysis, both Robinson et al. [13] and McConell et al. [8] observed in 16 subjects (50\% of the total sample) that high rates of fluid consumption during vigorous $1 \mathrm{~h}$ cycling exercise were associated with abdominal bloating and decrements in cycling performance. Therefore, we speculate that a fluid intake rate greater than the rate of gastric emptying (or intestinal absorption) during vigorous intensity cycling of $1 \mathrm{~h}$ may impair performance as a result of the associated gastrointestinal distress and overall discomfort. 'However, fluid intake rates equal to or substantially lower than gastric emptying, or even mouth rinsing, may have produced different results. Moreover, as demonstrated by Below et al. [25], ingesting fluid at a high rate may not be deleterious to all athletes during $1 \mathrm{~h}$ cycling performance.

In the present meta-analysis, the optimal rates of fluid intake for moderate intensity cycling of durations $>1-\leq 2 \mathrm{~h}(0.15-0.20 \mathrm{~mL} / \mathrm{kg}$ body mass/min) and $>2 \mathrm{~h}$ (ad libitum or $0.14-0.27 \mathrm{~mL} / \mathrm{kg}$ body mass/min) were lower than those observed in durations of $1 \mathrm{~h}$ but sufficient to limit dehydration and promote performance improvements of at least $2 \%$ and $3 \%$, respectively. Therefore, it is not clear if the effect on ECP was due to the duration and intensity of exercise or due to the intake of fluid. If fluid intake was the primary reason, it may have contributed to improving ECP through decreased cardiovascular and thermal strain [26], thirst sensation [4] or a potential placebo effect as participants were not blinded to oral rehydration [27]. Interestingly, all but one (ad libitum drinking) of these rates of fluid consumption were sufficient to maintain the loss of body mass $\leq 2 \%$, which 
supports the contention of Cheuvront et al. [26] that the goal of drinking during exercise is to prevent a body mass loss $>2 \%$.

As depicted in Figure 8c, drinking fluid at a rate dictated by thirst offered the greatest performance benefit during a cycling task $>2 \mathrm{~h}$. This observation matches that of Backes and Fitzgerald [28] who recently demonstrated that ad libitum drinking improves running endurance capacity, compared with planned drinking. However, other studies have shown that ad libitum drinking does not further improve cycling [9] or running performance [29-31], compared with prescribed drinking aimed at limiting dehydration. Both drinking to thirst and planned fluid intake are viable strategies and their use should be based on the cyclist's preference, environment, course, type and duration of event, rules and opportunities to drink. In certain situations a cyclist may be able to plan and strategize fluid intake, whilst in others, using thirst and the opportunity to drink may be more appropriate. Based on the limited available evidence from the present meta-analysis, we cannot conclusively conclude that one strategy is superior to the other and athletes are encouraged to adjust fluid intake in accordance with the demands of the event. Mathematical assimilation of the findings from several studies can provide additional insight, but it also has the potential to distort results if not considered in the context of the practicalities of sport performance and competition. In the event that a cyclist has not, or cannot plan fluid intake for a given exercise task and that drinking to thirst is not an option, the evidence-based fluid intake recommendations offered by this meta-analysis provide initial guidelines. However, this needs to take into account the possible pattern of fluid replacement during exercise, which is highly dependent on the opportunity for cyclists to drink. Indeed, during cycling events the loss of aerodynamics, bike control, race tempo, gastrointestinal comfort or fluid collection from designated stations are considerable factors that impact when a cyclist may consume fluid. Studies are needed to examine the intricate relationship between rate of drinking, pattern of drinking and frequency of drinking on ECP.

For those studies that showed performance benefits for cycling exercises $>1 \mathrm{~h}-\leq 2 \mathrm{~h}$ and $>2 \mathrm{~h}$ the average rate of fluid intake was 0.17 (range: $0.15-0.20$ ) and 0.20 (range: $0.14-0.32$ ) mL/kg body 
mass/min, respectively. Ross et al. [32] collected data during the Tours of Gippsland and Geelong and observed that cyclists ingested fluid at a rate of $\sim 0.09-0.13 \mathrm{~mL} / \mathrm{kg}$ body mass $/ \mathrm{min}$ during road races of, on average, $>1-\leq 2 \mathrm{~h}$ in duration. This rate of fluid intake is somewhat lower than the rates at which the laboratory-based studies included in this meta-analysis provided fluid. However, it must be noted that both Tours were held at ambient temperatures of $\sim 15^{\circ} \mathrm{C}$ which, at least in part, may explain the relatively low rate of fluid intake observed. Nevertheless, it remains to be determined whether the recommendation to drink at a rate of $0.15-0.20 \mathrm{~mL} / \mathrm{kg}$ body mass $/ \mathrm{min}$ during a cycling task of $>1 \mathrm{~h}-\leq 2 \mathrm{~h}$ can be applied to real-world cycling conditions. On the other hand, studies [33-35] which examined the rate of drinking of cyclists during outdoor road races $>2 \mathrm{~h}$ report rates of fluid intakes of $\sim 0.09-0.30 \mathrm{~mL} / \mathrm{kg}$ body mass $/ \mathrm{min}$, which is in line with what individuals were asked to drink in studies included in the present meta-analysis. Agreement between laboratory- and field-based observations provide evidence that the fluid intake recommendation for cycling exercise $>2 \mathrm{~h}$ can be confidently transferred to outdoor exercise conditions.

It is important that the recommendations arising from the current meta-analysis be interpreted and applied in the appropriate context. First, the influence of the rate of fluid intake on ECP is limited to nine studies with variations in duration, intensity and rehydration strategies. Second, the incompleteness of literature related to fluid intake and cycling performance creates an inability to clearly identify the benefits of consuming realistic volumes of fluid during cycling events of $1 \mathrm{~h}$ in duration. Third, results of the present meta-analysis are pertinent to the reality of endurance cycling only and should not be transposed to other sports or modes of exercise. Fourth, they are relevant for competitive cyclists under racing or training conditions where peak performance is sought. Although they may potentially also apply to low intensity training conditions, one should realize that studies from which they derive were not designed within this context. Fifth, it is unclear whether they can be extended to women since they represented only $10 \%$ of the population of subjects studied in the present meta-analysis. Along the same line, the results of the present meta- 
analysis do not apply to younger ( $<18$ y) and masters ( $>50$ y) cyclists. Sixth, the present results do not apply to cyclists who commence exercise in a hypohydrated state. Finally, findings are valid for cycling conditions where ambient temperatures range from $20-33^{\circ} \mathrm{C}$.

\section{Conclusion}

The effect of fluid consumption on ECP is complex and needs to be considered in the context of the specific scenario in which it is applied. Based on the available literature, the results of the present meta-analysis show that consuming fluid at high rates $(0.15-0.34 \mathrm{~mL} / \mathrm{kg}$ body mass $/ \mathrm{min})$ during high intensity $1 \mathrm{~h}$ cycling exercise conducted between $20-33^{\circ} \mathrm{C}$ is not conducive to ECP. In contrast, moderate intensity ECP may be improved under temperatures of $20-33^{\circ} \mathrm{C}$ when fluid is consumed at a rate of $0.15-0.20 \mathrm{~mL} / \mathrm{kg}$ body mass $/ \mathrm{min}$ and ad libitum or between $0.14-0.27 \mathrm{~mL} / \mathrm{kg}$ body mass/min for exercise durations of $>1-2 \mathrm{~h}$ and $>2 \mathrm{~h}$, respectively. These fluid intake recommendations offer further options for cyclists who do not use personalized hydration plans or follow thirst as a way to gauge fluid replacement during exercise. Further work is needed to examine a more authentic view of cycling hydration during high intensity, $1 \mathrm{~h}$ performance.

\section{COMPLIANCE WITH ETHICAL STANDARDS}

\section{Funding}

No sources of funding were used to assist in the preparation of this article.

\section{Conflicts of Interest}

Justin Holland, Tina Skinner, Christopher Irwin, Michael Leveritt and Eric D.B. Goulet declare that they have no conflicts of interest relevant to the content of this review. 


\section{REFERENCES}

1. Goulet ED. Effect of exercise-induced dehydration on time-trial exercise performance: a metaanalysis. Br J Sports Med. 2011;45(14):1149-56.

2. Goulet ED. Effect of exercise-induced dehydration on endurance performance: evaluating the impact of exercise protocols on outcomes using a meta-analytic procedure. $\mathrm{Br} J$ Sports Med. 2013;47(11):679-86.

3. Sawka MN, Burke LM, Eichner ER, et al. American College of Sports Medicine position stand. Exercise and fluid replacement. Med Sci Sports Exerc. 2007;39(2):377-90.

4. Sawka MN, Noakes TD. Does dehydration impair exercise performance? Med Sci Sports Exerc. 2007;39(8):1209-17.

5. Arnaoutis G, Kavouras SA, Christaki I, et al. Water ingestion improves performance compared with mouth rinse in dehydrated subjects. Med Sci Sports Exerc. 2012;44(1):175-9.

6. Bachle L, Eckerson J, Albertson L, et al. The effect of fluid replacement on endurance performance. J Strength Cond Res. 2001;15(2):217-24.

7. Kay D, Marino FE. Failure of fluid ingestion to improve self-paced exercise performance in moderate-to-warm humid environments. J Therm Biol. 2003;28(1):29-34.

8. McConell GK, Stephens TJ, Canny BJ. Fluid ingestion does not influence intense 1-h exercise performance in a mild environment. Med Sci Sports Exerc. 1999;31(3):386-92.

9. Dugas JP, Oosthuizen $U$, Tucker $R$, et al. Rates of fluid ingestion alter pacing but not thermoregulatory responses during prolonged exercise in hot and humid conditions with appropriate convective cooling. Eur J Appl Physiol. 2009;105(1):69-80.

10. McConell GK, Burge CM, Skinner SL, et al. Influence of ingested fluid volume on physiological responses during prolonged exercise. Acta Physiol Scand. 1997;160(2):149-56.

11. Goulet ED. Dehydration and endurance performance in competitive athletes. Nutr Rev. 2012;70 Suppl 2:S132-6.

12. Moher D, Liberati A, Tetzlaff J, et al. Preferred reporting items for systematic reviews and metaanalyses the PRISMA statement. PLoS Med. 2009;6(7).

13. Robinson TA, Hawley JA, Palmer GS, et al. Water ingestion does not improve 1-h cycling performance in moderate ambient temperatures. Eur J Appl Physiol Occup Physiol. 1995;71(2-3):153-60.

14. Walsh RM, Noakes TD, Hawley JA, et al. Impaired high-intensity cycling performance time at low levels of dehydration. Int J Sports Med. 1994;15(7):392-8.

15. Maughan RJ, Shirreffs SM, Leiper JB. Errors in the estimation of hydration status from changes in body mass. J Sports Sci. 2007;25(7):797-804.

16. Maughan RJ, Bethell LR, Leiper JB. Effects of ingested fluids on exercise capacity and on cardiovascular and metabolic responses to prolonged exercise in man. Exp Physiol. 1996;81(5):847-59.

17. Maughan RJ, Fenn CE, Leiper JB. Effects of fluid, electrolyte and substrate ingestion on endurance capacity. Eur J Appl Physiol Occup Physiol. 1989;58(5):481-6.

18. Lipsey M, Wilson D. Practical meta-analysis: Sage Publications; 2000.

19. Follmann $D$, Elliott $P$, Suh $I$, et al. Variance imputation for overviews of clinical trials with continuous response. J Clin Epidemiol. 1992;45(7):769-73.

20. Hopkins WG. Calculating likely (confidence) limits and likelihoods for true values (Excel spreadsheet). In: A new view of statistics. sportsci.org2002 [Available from: sportsci.org/resource/stats/xcl.xls.

21. Borenstein M, Hedges LV, Higgins JPT, et al. Introduction to meta-analysis: Wiley; 2009.

22. Backx K, Howatson G, van Someren KA. Fluid ingestion strategies of competitive cyclists during 40 km time trial competition. J Sports Sci Med. 2007;6(4):572-3.

23. Costill DL, Saltin B. Factors limiting gastric emptying during rest and exercise. J Appl Physiol. 1974;37(5):679-83.

24. Mitchell JB, Voss KW. The influence of volume on gastric emptying and fluid balance during prolonged exercise. Med Sci Sports Exerc. 1991;23(3):314-9. 
25. Below PR, Mora-Rodriguez R, Gonzalez-Alonso J, et al. Fluid and carbohydrate ingestion independently improve performance during $1 \mathrm{~h}$ of intense exercise. Med Sci Sports Exerc. 1995;27(2):20010.

26. Cheuvront SN, Carter R, 3rd, Sawka MN. Fluid balance and endurance exercise performance. Curr Sports Med Rep. 2003;2(4):202-8.

27. Wall B, Watson G, Peiffer J. Current hydration guidelines are erroneous: dehydration does not impair exercise performance in the heat. Br J Sports Med. 2013;0:1-8.

28. Backes TP, Fitzgerald K. Fluid consumption, exercise, and cognitive performance. Biol Sport. 2016;33(3):291-6.

29. Dion T, Savoie FA, Asselin A, et al. Half-marathon running performance is not improved by a rate of fluid intake above that dictated by thirst sensation in trained distance runners. Eur J Appl Physiol. 2013;113(12):3011-20.

30. Lee MJ, Hammond KM, Vasdev A, et al. Self-selecting fluid intake while maintaining high carbohydrate availability does not impair half-marathon performance. Int J Sports Med. 2014;35(14):121622.

31. Lopez RM, Casa DJ, Jensen KA, et al. Comparison of two fluid replacement protocols during a 20-km trail running race in the heat. J Strength Cond Res. 2016;30(9):2609-16.

32. Ross ML, Stephens B, Abbiss CR, et al. Fluid balance, carbohydrate ingestion, and body temperature during men's stage-race cycling in temperate environmental conditions. Int J Sports Physiol Perform. 2014;9(3):575-82.

33. Armstrong LE, Johnson EC, McKenzie AL, et al. Endurance cyclist fluid intake, hydration status, thirst, and thermal sensations: gender differences. Int J Sport Nutr Exerc Metab. 2016;26(2):161-7.

34. Ebert TR, Martin DT, Stephens B, et al. Fluid and food intake during professional men's and women's road-cycling tours. Int J Sports Physiol Perform. 2007;2(1):58-71.

35. Hew-Butler T, Dugas JP, Noakes TD, et al. Changes in plasma arginine vasopressin concentrations in cyclists participating in a 109-km cycle race. Br J Sports Med. 2010;44(8):594-7. 
Table 1. Exercise protocol characteristics, rates of fluid intake and hypohydration levels of included research manuscripts and individual studies.

\begin{tabular}{|c|c|c|c|c|c|c|c|}
\hline $\begin{array}{l}\text { References } \\
\text { (alphabetical } \\
\text { order) }\end{array}$ & $\begin{array}{l}\text { Number of participants, sex and } \\
\text { age (yrs) }\end{array}$ & Exercise protocol & $\begin{array}{l}\text { Exercise duration } \\
(\mathbf{m i n}) \\
\text { and intensity } \\
\left(\% \dot{\mathbf{V}} \text { O }_{2 p e a k}\right)\end{array}$ & $\begin{array}{l}\text { Ambient } \\
\text { temperature }\left({ }^{\circ} \mathrm{C}\right)\end{array}$ & $\begin{array}{l}\text { Relative } \\
\text { humidity } \\
\text { (\%) }\end{array}$ & $\begin{array}{l}\text { Fluid intake } \\
\text { (mL/min and } \\
\text { mL/kg body } \\
\text { mass/min) }\end{array}$ & $\begin{array}{l}\text { Dehydration level } \\
\text { (\% body mass) }\end{array}$ \\
\hline \multicolumn{8}{|c|}{ Exercise duration of $1 \mathrm{~h}$} \\
\hline $\begin{array}{l}\text { Bachle et al. } \\
{[6]}\end{array}$ & $\begin{array}{l}10 \text { recreationally-trained } \\
\text { participants } 4 \text { men } 6 \text { women } \\
29 \pm 6\end{array}$ & $\begin{array}{l}\text { Fixed-duration } \\
\text { self-paced } \\
\text { time-trial }\end{array}$ & 60 and 61 & 21 & 72 & 20 and 0.30 & $\begin{array}{l}\text { NFI group: }-1.0 \text {; } \\
\text { FI group: +0.8 }\end{array}$ \\
\hline Kay and & 7 healthy cyclists 6 men 1 & Fixed-duration & 60 and 80 & Moderate: FI & 63 & Moderate: & A*. Moderate: \\
\hline Marino [7] & woman $21 \pm 3$ & $\begin{array}{l}\text { self-paced } \\
\text { time-trial }\end{array}$ & & $\begin{array}{l}\text { group vs. NFI } \\
\text { group: 19.8; } \\
\text { Warm: FI } \\
\text { group vs. NFI } \\
\text { group: } 33.2\end{array}$ & & $\begin{array}{l}22 \text { and } 0.29 \\
\text { Warm: } \\
25 \text { and } 0.34\end{array}$ & $\begin{array}{l}\text { NFI group: -1.8; } \\
\text { FI group: } 0 \\
\text { B*. Warm: } \\
\text { NFI group: -2.2; } \\
\text { FI group: } 0\end{array}$ \\
\hline McConell et & 8 well-trained cyclists and & 1. Fixed- & 1: 45 and 85 & 21 & 41 & FR-50 & 1A*. FR-50: \\
\hline
\end{tabular}




\begin{tabular}{|c|c|c|c|c|c|c|c|}
\hline \multirow[t]{5}{*}{ al. [8] } & triathletes 8 men 0 women 26 & intensity & \multicolumn{3}{|l|}{ 2: 15 and 81} & 12 and 0.15 & NFI group: -1.9; \\
\hline & \multirow[t]{4}{*}{ \pm 3} & + & & & & FR-100 & FI group: -1.0 \\
\hline & & 2. Fixed- & & & & 25 and 0.31 & $1 \mathrm{~B}^{*} . \mathrm{FR}-100$ \\
\hline & & duration time- & & & & & NFI group: -1.9; \\
\hline & & trial & & & & & FI group: 0.0 \\
\hline Robinson et & 8 endurance-trained & Fixed-duration & 60 and 85 & 20 & 60 & 25 and 0.32 & NFI group: -2.3; \\
\hline \multirow[t]{2}{*}{ al. [13] } & participants 8 men 0 women & self-paced & & & & & \multirow[t]{2}{*}{ FI group: -0.9} \\
\hline & $26 \pm 4$ & time-trial & & & & & \\
\hline \multirow[t]{4}{*}{ Mean \pm SD } & \multirow[t]{4}{*}{$8 \pm 1$} & \multirow[t]{4}{*}{---} & $60 \pm 0$ & \multirow[t]{4}{*}{$23 \pm 5$} & \multirow[t]{4}{*}{$57 \pm 13$} & $21 \pm 5$ & NFI group: \\
\hline & & & \multirow[t]{3}{*}{ and $78 \pm 10$} & & & and & $-1.9 \pm 0.5$ \\
\hline & & & & & & $0.29 \pm 0.07$ & FI group: \\
\hline & & & & & & & $-0.2 \pm 0.7$ \\
\hline \multicolumn{8}{|c|}{ Exercise duration $>1 \mathrm{~h}-2 \mathrm{~h}$} \\
\hline Maughan et & 12 healthy participants 12 & Fixed-intensity & 93.5 and 70 & 21 & 21 & 11 and 0.16 & NFI group: -1.9; \\
\hline al. [16] & men 0 women $24 \pm 3$ & to exhaustion & & & & & FI group: -0.6 \\
\hline
\end{tabular}




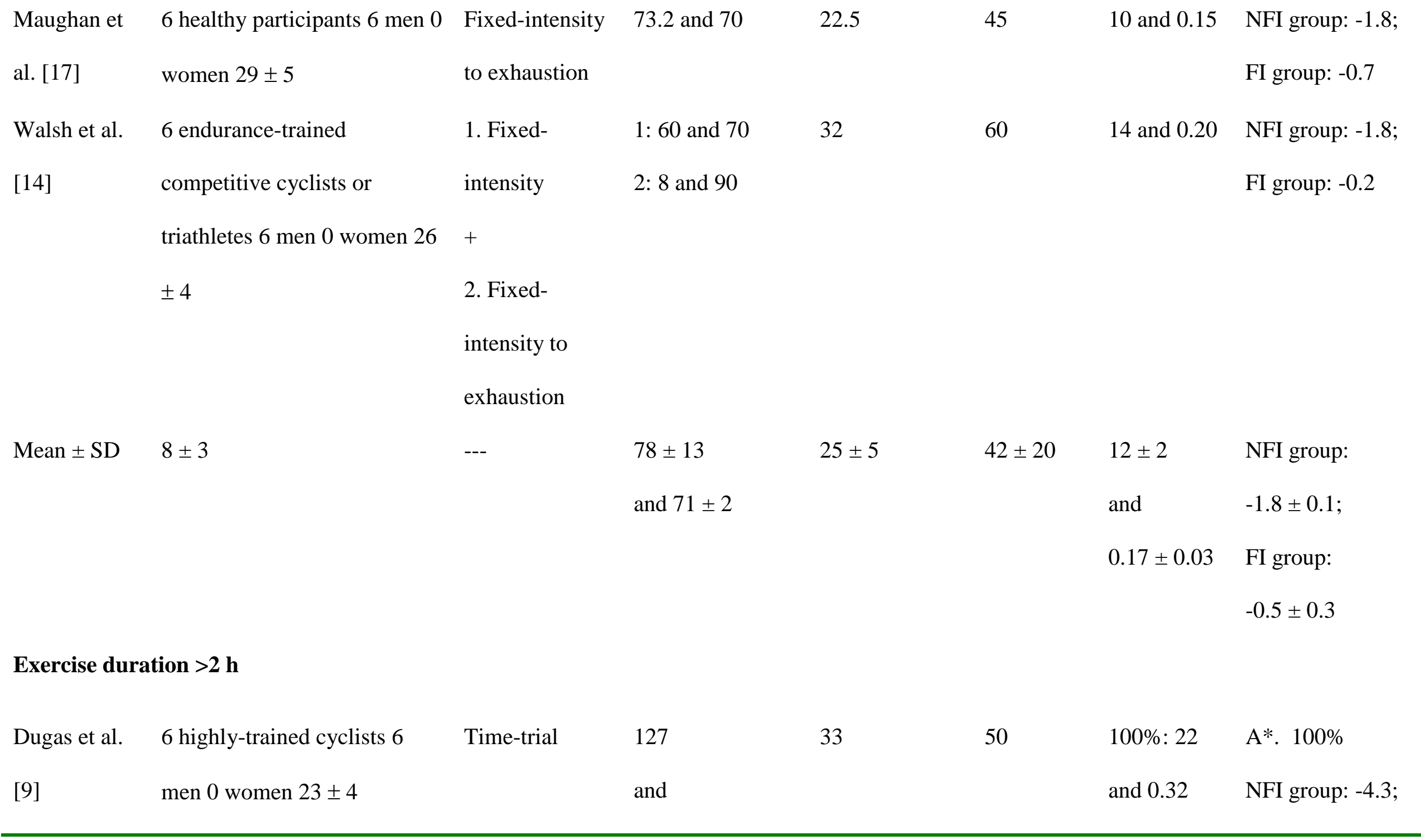


and 0.21

B*. $66 \%$

33\%: 7 an

0.10

Ad lib: 11

and 0.16

$$
\text { NFI group: -4.3; }
$$

FI group: -2.9

D*. Ad lib

NFI group: -4.3;

FI group: -2.1

McConell et 7 well-trained cyclists and

al. [10] triathletes 7 men 0 women 24

$\pm 3$
2. Fixed-

intensity to

exhaustion

1: 120 and 70

2: 4 and 90

21

$---$
$126 \pm 2$
43

FR-50:

9 and 0.14

FR-100:

19 and 0.27

B*. FR-100

NFI group: -3.2;

FI group: -0.1

$29 \pm 6$
$48 \pm 4$
NFI group: 
$-1.6 \pm 1.1$

Ad lib: Ad libitum drinking; FI: Fluid intake group; FR-50: 50\% fluid replacement; FR-100: 100\% fluid replacement; NFI: No fluid intake group;

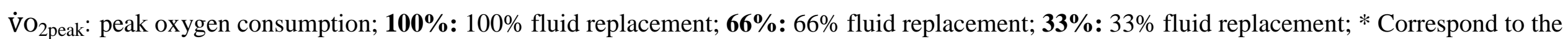
studies found in the Forest Plot. 


\section{Figure legends}

\section{Figure 1.}

Flowchart demonstrating the selection process used for the inclusion and exclusion of articles for this meta-analysis.

\section{Figure 2.}

Forest plot demonstrating the impact of fluid intake on overall cycling performance. CI: confidence interval. $\$$ : represents the overall weighted mean treatment effect. Sizes of squares are proportional to the weight of the study. References and letters in brackets match those found in Table 1.

\section{Figure 3.}

Correlations between the change in power output and the rate of fluid intake (a), exercise duration (b) and exercise intensity (c). Diameters of circles are proportional to the weight of the study. $\dot{\mathrm{V}} \mathrm{O}_{2 \text { peak: }}$ peak oxygen consumption.

\section{Figure 4.}

Correlations between the change in power output and temperature (a) and humidity level (b). Diameters of circles are proportional to the weight of the study.

\section{Figure 5.}

Correlations between the change in power output and rate of fluid intake, while taking into account exercise duration. Diameters of circles are proportional to the weight of the study.

O: Exercise duration of $1 \mathrm{~h}$; : Exercise duration $>1 \mathrm{~h}-\leq 2 \mathrm{~h} ; \mathrm{c}:$ : Exercise duration $>2 \mathrm{~h}$.

\section{Figure 6.}

Correlations between the prevention of body mass loss and the \% change in power output (a) and the fluid intake-associated hypohydration level and the \% change in power output (b), while taking into account exercise duration. Diameters of circles are proportional to the weight of the study. O: Exercise duration of $1 \mathrm{~h}$; : Exercise duration $>1 \mathrm{~h}-\leq 2 \mathrm{~h}$; 5 : Exercise duration $>2 \mathrm{~h}$. 


\section{Figure 7.}

Forest plots demonstrating the impact of fluid intake on cycling performance during exercises of $1 \mathrm{~h}$ (a), $>1-\leq 2 \mathrm{~h}$ (b) and $>2 \mathrm{~h}$ (c) in duration. CI: confidence interval. $\checkmark$ : represents the overall weighted mean treatment effect. Sizes of squares are proportional to the weight of the study. References and letters in brackets match those found in Table 1.

\section{Figure 8.}

Rates of fluid intake associated with changes in cycling performance for exercises of $1 \mathrm{~h} \mathrm{(a),}>1-\leq 2$

h (b) and $>2 \mathrm{~h}$ (c) in duration, while taking into account the change in power output and the corresponding fluid intake-associated hypohydration level.

$\longleftrightarrow$ : represents the threshold for performance gain/decrement.

$4--\rightarrow$ : represents a threshold loss of body mass of $2 \%$.

: represents the range of rate of fluid intake associated with most performance gains.

๑: represents the change in power output.

$\square$ : represents the fluid intake-associated hypohydration level.

: represents the relationship between fluid intake and the change in performance.

: represents the relationship between the rate of fluid intake and the fluid intake-associated

hypohydration level. 


\section{Figures.}

Figure 1.

Articles identified through database searching $(N=15,532)$

Articles screened for duplicates ( $\mathrm{N}=2,787$ eliminated)

Articles remaining after duplicate removal $(\mathrm{N}=12,745)$

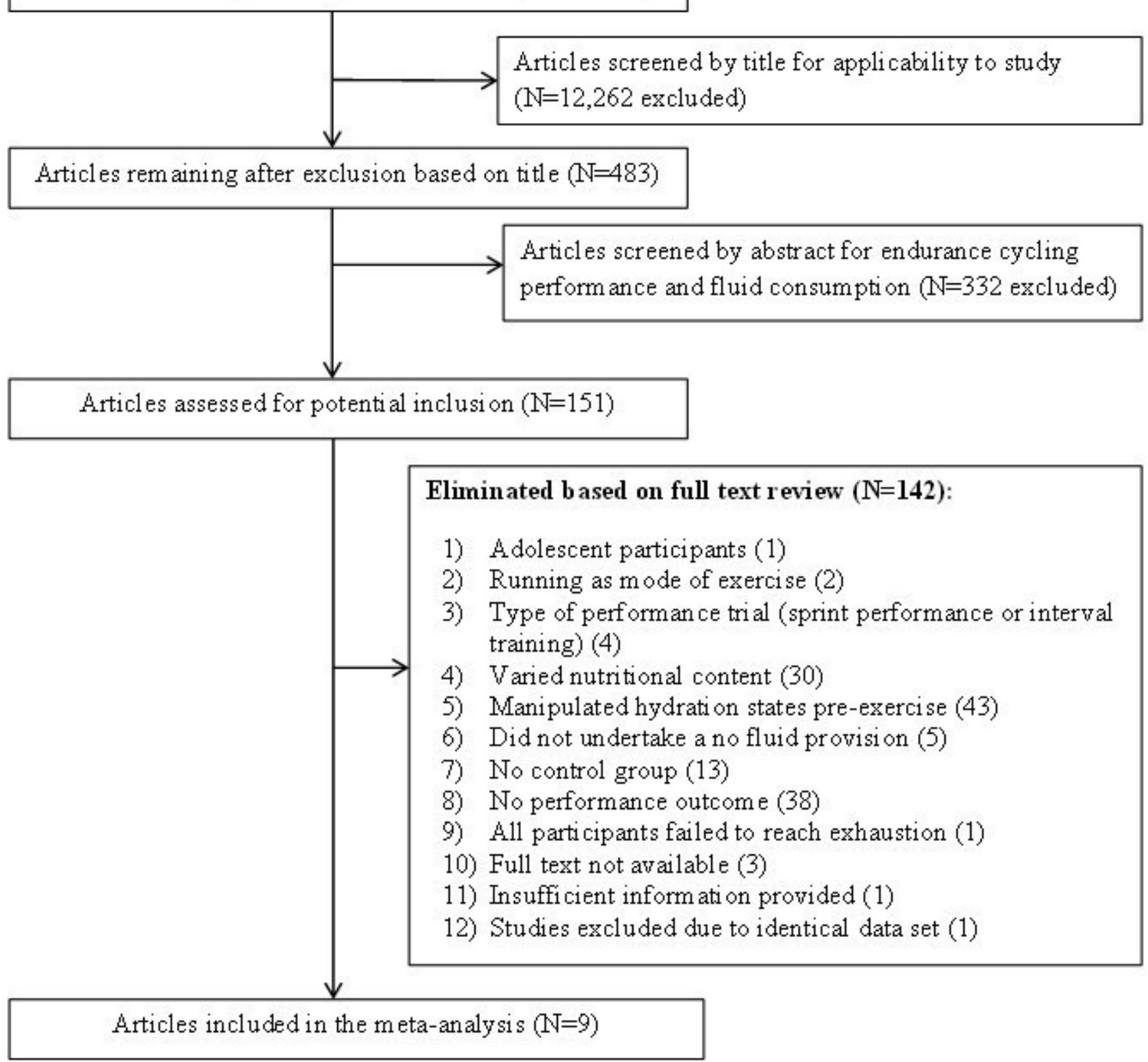


Figure 2.

References

Kay and Marino [7] [A]

Robinson et al. [13]

Dugas et al. [9] [C]

McConell et al. [8] [1A]

McConell et al. [8] [1B]

Kay and Marino [7] [B]

Maughan et al. [17]

Maughan et al. [16]

McConell et al. [10] [2A]

Bachle et al. [6]

Walsh et al. [14]

Dugas et al. [9] [B]

Dugas et al. [9] [A]

McConell et al. [10] [2B]

Dugas et al. [9] [D]
Statistics for each study

\section{Difference Standard Lower Upper} in means error

limit limit $p$-Value

$\begin{array}{ccccc}-7.66 & 5.88 & -19.18 & 3.86 & 0.19 \\ -3.30 & 1.40 & -6.04 & -0.56 & 0.02 \\ -2.85 & 2.12 & -7.01 & 1.31 & 0.18 \\ -2.21 & 1.20 & -4.56 & 0.14 & 0.07 \\ -1.47 & 2.41 & -6.19 & 3.25 & 0.54 \\ 0.00 & 5.57 & -10.92 & 10.92 & 1.00 \\ 0.78 & 0.91 & -1.00 & 2.56 & 0.39 \\ 1.52 & 0.69 & 0.17 & 2.87 & 0.03 \\ 3.20 & 3.26 & -3.19 & 9.59 & 0.33 \\ 3.25 & 7.19 & -10.84 & 17.34 & 0.65 \\ 3.61 & 0.76 & 2.12 & 5.10 & 0.00 \\ 5.25 & 5.07 & -4.69 & 15.19 & 0.30 \\ 6.32 & 5.48 & -4.42 & 17.06 & 0.25 \\ 6.32 & 2.58 & 1.26 & 11.38 & 0.01 \\ 9.24 & 2.93 & 3.50 & 14.98 & 0.00 \\ 1.08 & 0.91 & -0.70 & 2.86 & 0.23\end{array}$

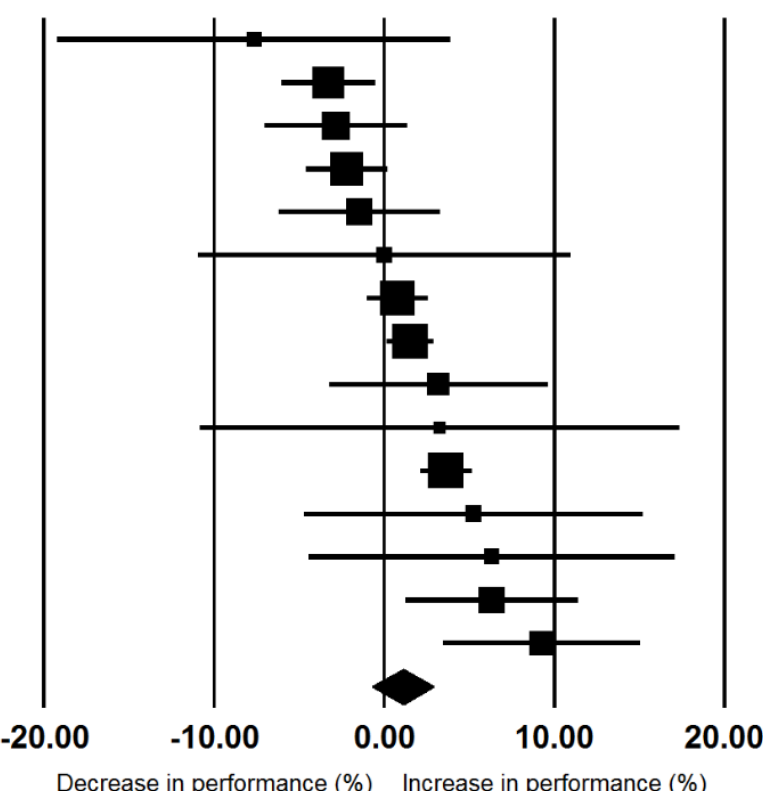


Figure 3.
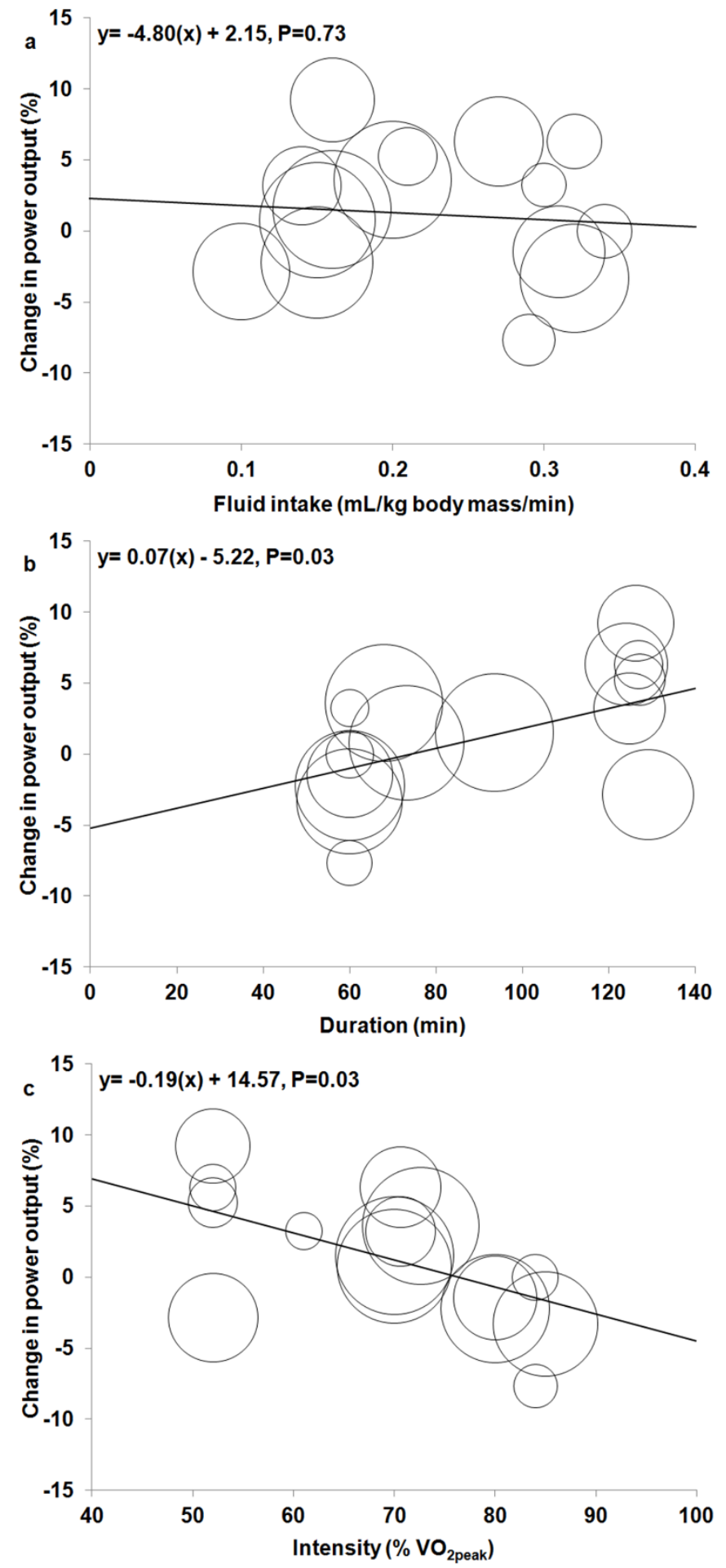
Figure 4.
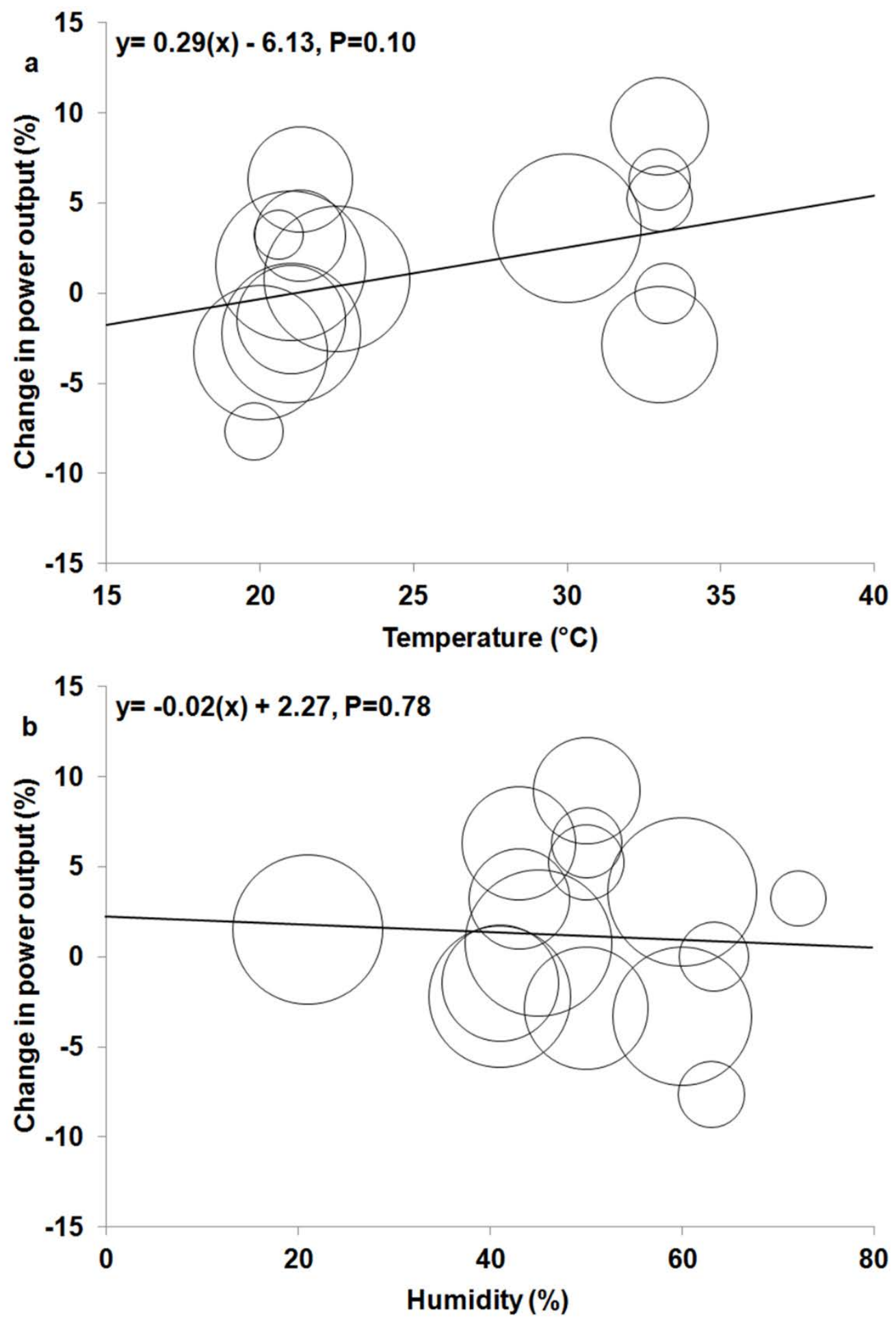
Figure 5.

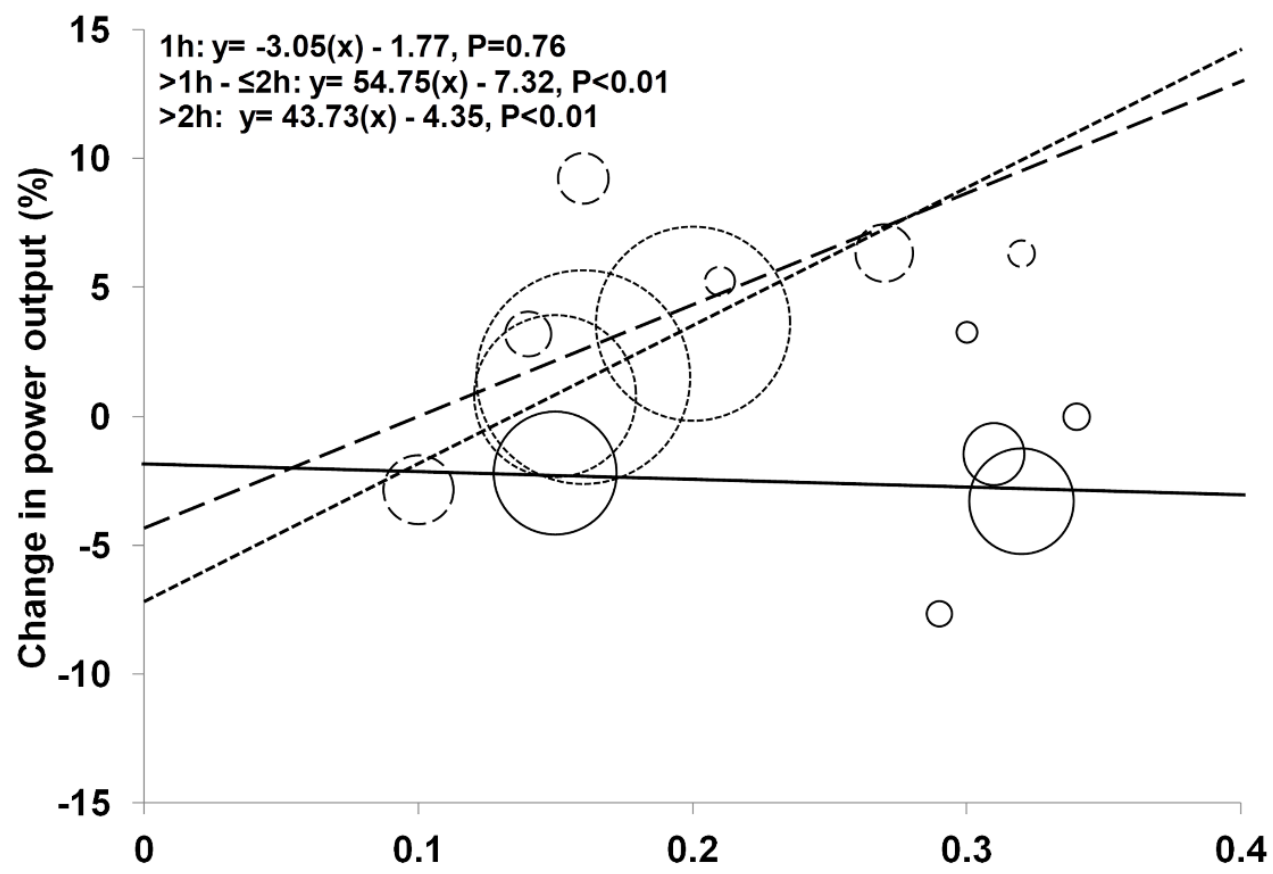

Fluid intake ( $\mathrm{mL} / \mathrm{kg}$ body mass/min) 
Figure 6.
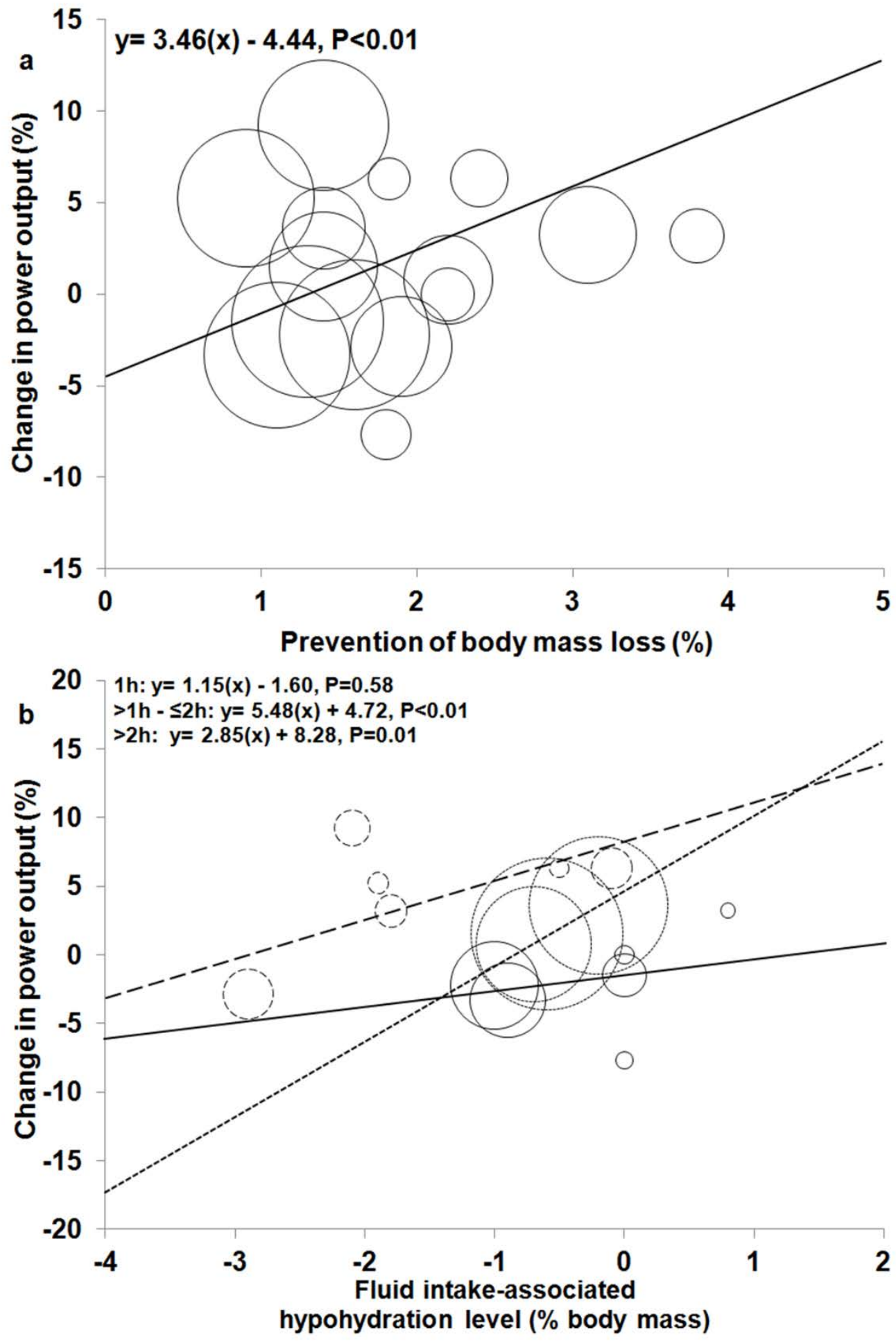
Figure 7.

a

References
Statistics for each study

Difference Standard Lower Upper

in means error limit limit $p$-Value

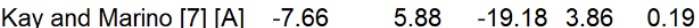

$\begin{array}{lllllll}\text { Robinson et al. [13] } & -3.30 & 1.40 & -6.04 & -0.56 & 0.02\end{array}$

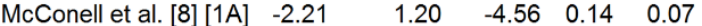

McConell et al. [8] [1B] $\quad-1.47 \quad 2.41 \quad-6.19 \quad 3.25 \quad 0.54$

Kay and Marino [7] [B] $\quad 0.00 \quad 5.57 \quad-10.92 \quad 10.92 \quad 1.00$

Bachle et al. [6] $\quad 3.25$

$-2.49$

$\begin{array}{llll}7.19 & -10.84 & 17.34 & 0.65\end{array}$

$\begin{array}{llll}0.83 & -4.11 & -0.87 & 0.00\end{array}$

Statistics for each study

b

References

Difference Standard Lower Upper

in means error limit limit p-Value

Maughan et al. [17] 0.78

Maughan et al. [16] 1.52

Walsh et al. [14] 3.61

0.91

$\begin{array}{lll}-1.00 & 2.56 & 0.39\end{array}$

$\begin{array}{llll}0.69 & 0.17 & 2.87 & 0.03\end{array}$

2.06

$\begin{array}{llll}0.76 & 2.12 & 5.10 & 0.00\end{array}$

$\begin{array}{llll}0.45 & 1.19 & 2.93 & 0.00\end{array}$

References

Statistics for each study

Difference Standard Lower Uppe

in means error limit limit $p$-Value

Dugas et al. [9] [C]

$-2.85$

McConell et al. [10] [2A] $\quad 3.20$

Dugas et al. [9] [B] $\quad 5.25$

Dugas et al. [9] [A]

5.25
6.32

McConell et al. [10] [2B] 6.32

Dugas et al. [9] [D]

9.24

3.21

$\begin{array}{cccc}2.12 & -7.01 & 1.31 & 0.18 \\ 3.26 & -3.19 & 9.59 & 0.33 \\ 5.07 & -4.69 & 15.19 & 0.30 \\ 5.48 & -4.42 & 17.06 & 0.25 \\ 2.58 & 1.26 & 11.38 & 0.01 \\ 2.93 & 3.50 & 14.98 & 0.00 \\ 1.24 & 0.79 & 5.64 & 0.01\end{array}$

$\begin{array}{llll}1.24 & 0.79 & 5.64 & 0.01\end{array}$

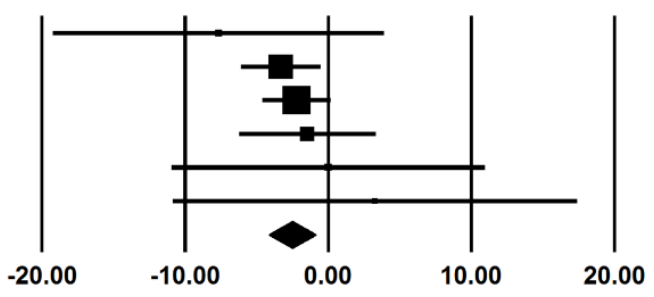

Decrease in performance (\%) Increase in performance (\%)

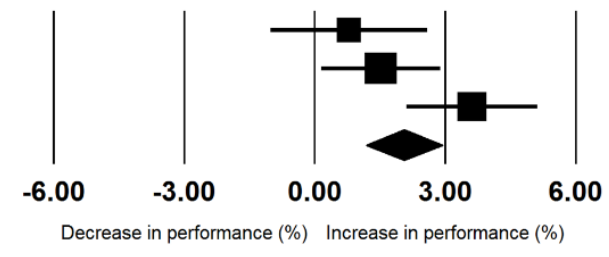

Difference in means and $95 \% \mathrm{Cl}$

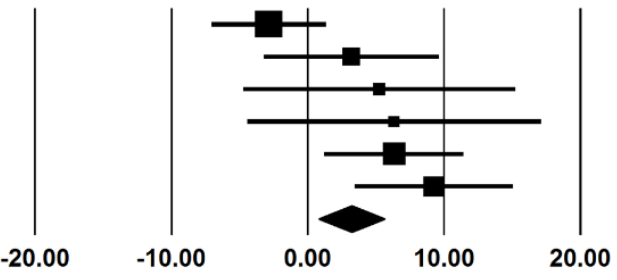

Decrease in performance (\%) Increase in performance (\%) 
Figure 8.
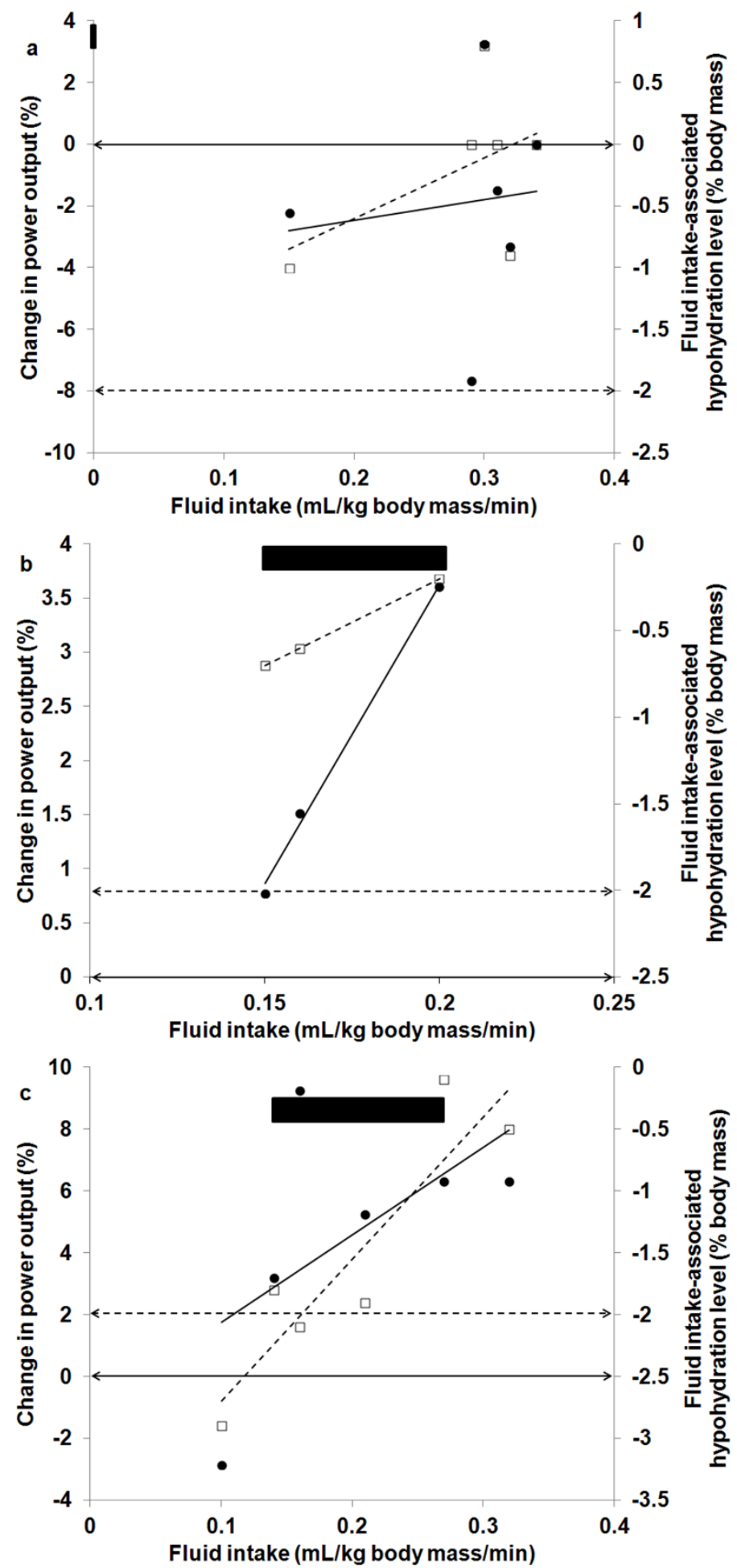